\title{
Radiation Dosimetry Using Three-Dimensional Optical Random Access Memories
}

\author{
Marko Moscovitcha, Gary W. Phillipsa,b, Brian M. Cullumc, Joel Mobleyc, \\ James S. Bogard ${ }^{\mathrm{c}}$, Dimitris Emfietzoglou ${ }^{\mathrm{d}}$ and Tuan Vo-Dinh ${ }^{\mathrm{c}}$ \\ a. Georgetown University School of Medicine, 3970 Reservoir Road NW, \\ Washington DC 20007, USA \\ b. U.S. Naval Research Laboratory, 4555 Overlook Avenue SW, \\ Washington DC 20375, USA \\ c. Oak Ridge National Laboratory, Bethel Valley Road, MS-6101, \\ P.O. Box 2008, Oak Ridge, TN, USA \\ d. University of Ioannina Medical School, 45110 Ioannina, Greece
}

\begin{abstract}
The ability to determine particle type and energy plays an important role in the dosimetry of heavy charged particles (HCP) and neutrons. A new approach to radiation dosimetry is presented, which is shown to be capable of particle type and energy discrimination. This method is based on utilizing radiation induced changes in the digital information stored on three-dimensional optical random access memories (3D ORAM). 3D ORAM is a small cube (a few $\mathrm{mm}^{3}$ ) composed of poly(methyl methacrylate) doped with a photochromic dye, and it was originally proposed as a memory device in high speed parallel computers. A Nd:YAG laser system is used to write and read binary information (bits) on the ORAM, which functions as a charged particle detector. Both the read and the write processes use two laser beams that simultaneously strike the material to cause a color change at their intersection (similar to the darkening of light-sensitive sunglasses when exposed to sunlight.) The laser produces color changes in the ORAM, which then reverts to the original color ("bit-flips") at sites where energy is deposited from interaction with incident HCP or neutron-recoil protons. The feasibility of this approach was demonstrated both theoretically and experimentally. Calculations based on track structure theory (TST) predict that when HCP interact with the ORAM material, the local energy deposition is capable of inducing measurable "bit-flips". These predictions were recently confirmed experimentally using two types of ORAM systems, one based on spirobenzopyran and the other on anthracene, as the photochromic dyes.
\end{abstract}

\section{Introduction}

Three-dimensional optical random access memory, which makes use of a bistable photochromic molecule such as spirobenzopyran (SP) embedded in a polymer matrix, has been developed for storage of large volumes of computer data [1]. Information is stored in a 3D array at the intersection of crossed laser beams using a two-photon excitation process (Figure 1) that converts the SP molecule from the more stable isomer on the left (representing a binary 'zero' bit) to the less stable isomer on the right (representing a binary 'one'.) The latter exhibits stimulated fluorescence upon absorption of sufficient 
energy. These properties allow the binary state to be written and read using crossed laser beams to address a specific 3D location. As computer memory it has a very high potential storage density of about one terabyte per cubic centimeter.

The use of this material for real-time dosimetry of neutrons or other energetic particles was first proposed by Moscovitch [2,3]. Theoretical calculations by Moscovitch and Emfietzoglou [4] indicate that the energy deposited along a track due to passage of a charged particle through this material results in an increase above background of the local de-excitation rate of the less stable isomer with track characteristics which depend on the particle type and energy. Thus, dosimeters based on these materials could provide detailed information on particle type and energy distribution for neutrons and heavy charged particles (HCP). This would allow more accurate radiation measurements for applications such as nuclear non-proliferation monitoring and space dosimetry.

A preliminary design for a 3D ORAM dosimetry reader has been described by Mobley et al. [5]. The 3D pattern of radiation exposure is read by crossed Nd:YAG laser beams and interpreted by computer using neural network techniques to reconstruct the incident radiation field from the size distribution of the particle tracks. If sufficient sensitivity can be achieved for real-time readout, the device could be used as a detector/dosimeter for environmental and non-proliferation applications. The effects of exposure of 3D ORAM materials to ionizing radiation have been measured and results were reported by Phillips et al. [6] and Readshaw et al. [7]. In the following sections we describe our results from the application of 3D ORAM materials to both HCP and neutron dosimetry.

\section{Heavy Charged Particle Dosimetry}

Thin films of PMMA were cast from solution with the SP molecule at concentrations of $0.1 \%$ and $1 \%$ by weight. These films were irradiated with uniform fluxes of protons, alpha particles and ${ }^{12} \mathrm{C}^{+3}$ ions at fixed energies from 0.6 to $6.5 \mathrm{MeV}$. The materials were exposed to fluences from $10^{10}$ to $10^{14} \mathrm{~cm}^{-2}$, resulting in doses of $10^{3}$ to $10^{7} \mathrm{~Gy}$ in the exposed volume of the films.

The exposures were made with the SP in the more stable geometric isomer (Figure 1, Form I) which is clear and non-fluorescent. In the absence of UV light, this form is stable at room temperature. The less stable isomer (Form II) is dark blue and fluoresces in the red. In PMMA it has a half-life for thermal de-excitation of about 30 minutes at room temperature, too short for convenient exposure to radiation and measurement of the effects.

Exposure of the SP doped PMMA films to HCP radiation resulted in an apparently permanent change in the SP molecule. The unexposed films were clear, but exposure resulted in a visible yellow beam spot which darkened with increasing fluence. Pure PMMA films exposed to similar fluences showed no yellowing of the beam spot (see Figure 2).

The exposed spots were examined under a BIORAD MRC-600 confocal laser scanning microscope (CLSM). With the CLSM it is possible to section the material optically in order to look at effects of the charged particle exposure versus depth in the film. Stimulated fluorescence was observed through an optical filter using a dual 
wavelength argon laser. The exposed beam spots were found to fluoresce under both 488 $\mathrm{nm}$ and $514 \mathrm{~nm}$ excitation. Fluorescence was observed for all exposures, even the lower fluences that did not produce a visible beam spot on the films, while adjacent unexposed spots on the same films did not fluoresce. Furthermore, the fluorescent intensity was undiminished after a period of several months. To rule out changes in the PMMA matrix, films without the SP molecule were also exposed at some of the higher fluences. No visible change was observed in these films and no fluorescence was observed under the CLSM.

Figure 3 shows CLSM profiles of intensity versus depth for $0.6 \mathrm{MeV}$ to $2.5 \mathrm{MeV}$ protons normalized to a maximum intensity of 200 (arbitrary units). These are plotted versus the relative depth obtained from the CLSM focal depth. The surface of the film is on the left side of the curves and the position of the left half-maximum is arbitrarily adjusted to zero depth. Clearly the depth of penetration in the film increases with the particle energy. The shape of these curves can be understood in terms of the CLSM response which is discussed below.

In Figure 4, the full-width at half-maximum (FWHM) of the depth profiles is plotted against energy per nucleon. Within the limited range in energy per nucleon measured here, the 0.5 and $1.25 \mathrm{MeV} / \mathrm{amu}$ alpha particles and the $0.54 \mathrm{MeV} / \mathrm{amu}{ }^{12} \mathrm{C}$ ions appear to lie on the same curve as the protons.

The width of the depth profiles is expected to depend on the range of the particles. Figure 5 shows the result of transport calculations for the range versus energy per nucleon for protons, alphas and ${ }^{12} \mathrm{C}$ ions in PMMA using the SRIM/TRIM code [8]. At $0.5 \mathrm{MeV} / \mathrm{amu}$ the ranges are nearly identical. At higher energies per nucleon the proton and alpha curves remain close while the curve for ${ }^{12} \mathrm{C}$ diverges.

The FWHM of the depth profiles in Figure 4 depends both on the range of the particles and on the properties of the CLSM instrument. Comparison of Figures 4 and 5 shows that the measured FWHM for protons is significantly less than the calculated range at the high energy end, 1.5-2.5 MeV. As discussed in the modeling results presented below, these differences are probably due to a lack of accuracy in the CLSM depth calibration and also to films which were too thin to stop the higher energy protons. At the low energy end, 0.5-0.6 MeV/amu, the measured FWHM is greater than the calculated range due to the finite depth resolution of the CLSM, which is expected to be poorer for an extended source than for a point source.

To see how the results of the exposures varied for the different particles and energies measured in this study, the fluorescent intensities from the CLSM analysis were compared at the depth of maximum intensity in each film. Figure 6 shows the maximum fluorescent intensities plotted versus the dose. For a given particle and energy, there is a broad dynamic range in which the fluorescent intensity increases with the dose before saturating at the higher doses. The variations of the data about the smooth curves are likely due to irregularities in the films or in the beam profiles.

The top three plots in Figure 6 are for films of PMMA doped with SP at the 1\% level, while the bottom three plots are for films doped at the $0.1 \%$ level. The $1 \%$ SP data are approximately a factor of 10 higher than the $0.1 \%$ data, showing that the effect depends on the SP concentration. An SP concentration of $1 \%$ is close to the practical limit due to molecular aggregation that occurs at higher concentrations. 
Figure 6 also shows that for a given particle, dose and SP concentration, particles with higher energy have an increased fluorescent effect on the films. The saturation at high doses occurs at different intensities for different particles and energies. At all doses, the intensity for $2.5 \mathrm{MeV}$ protons is significantly greater than for $0.6 \mathrm{MeV}$ protons and similarly the intensity for $5 \mathrm{MeV}$ alphas is significantly greater than for $2 \mathrm{MeV}$ alphas. In general the intensity at a given dose increases with energy per nucleon and decreases with the linear energy transfer (LET) calculated from the SRIM/TRIM code. This decrease with LET is probably due to saturation effects for the conversion of the SP molecule from Form I to a new species (distinct from Form II) in a process analogous to that calculated by Moscovitch and Emfietzoglou [4] for the transformation from Form II to Form I. They calculate a "bit-flip probability" as a function of exposure, which is seen to saturate at a probability of 1 . Once saturation is complete, any additional energy deposited along the track is "wasted." Because of this saturation, the observed effect of high LET particles is less than that of lower LET particles with the same energy per nucleon.

\section{3D ORAM Materials Applied to Neutron Detection}

The use of 3D ORAM materials as neutron dosimeters has been investigated by Cullum et al. [9] using a two photon crossed laser readout system for measuring the fluorescence in bulk materials. Neutrons do not leave a track in these materials, but they do have a significant probability of scattering off the nucleus of a hydrogen atom, producing an energetic proton whose track can then be detected. The mean free path of fast neutrons in the $\mathrm{MeV}$ energy range is several millimeters so that bulk materials with thicknesses of a centimeter or more are required to detect the neutrons, unlike the case for charged particles, considered in the previous section, where thin films of the order of $100 \mu \mathrm{m}$ were sufficient to stop the particles. For commonly used neutron dosimetry techniques such as thermoluminescent dosimetry (TLD), their sensitivity is strongly dependent on the energy spectrum of the neutrons and thus the dosimeters must be calibrated with a known neutron energy field in order to achieve high accuracy. When the energy field is not well known, large errors are possible. The previous section showed that the charged particle type and energy could be determined from the track profile. Thus it should be possible to determine the neutron energy field from measuring the characteristics of tracks left by the recoil charged particles in the materials. Cullum et al [9] have taken the first steps toward a practical 3D ORAM neutron dosimeter by demonstrating sensitivity to neutrons and by developing a prototype cross-beam laser readout system.

As discussed in the previous sections, 3D ORAM materials make use of a bistable photochromic molecule embedded in a polymer. The molecule is non-fluorescent in its most stable configuration (Form I, Figure 1), but can be excited by exposure to intense ultraviolet light into a less stable form (Form II) which fluoresces upon stimulation by two-photon absorption using a crossed laser beam technique. Two photochromic molecules, spirobenzopyran and anthracene were investigated. However, the fluorescent form of SP was found to be too unstable at room temperature for use as a practical dosimeter, whereas anthracene was shown to have less than $1 \%$ spontaneous conversion 
back to the non-fluorescent form after three months storage at room temperature [9]. Therefore the study of neutron detection focused on anthracene.

The basic anthracene molecule has a three-benzene-ring structure (the monomer) as shown in Figure 7. Following polymerization of the anthracene doped PMMA, the anthracene molecules typically reside in the dimeric form, consisting of two linked monomers. Model calculations yield two energetically favorable three dimensional geometries for the anthracene dimer (Figure 8), with the two monomers in crossed and displaced parallel configurations [10]. The geometric form of the dimer in anthracene doped PMMA has not been determined. In PMMA the dimeric form is non-fluorescent but can be excited to the fluorescent monomeric form by absorption of two 532-nm photons (or by a single 355-nm photon in the UV range) (Figure 9).

The effects of neutron irradiation on 1\% anthracene-doped PMMA were investigated by conversion of two identically prepared samples to the fluorescent form using UV light. One sample was stored in a radiation-free environment while the other was exposed to neutrons from a ${ }^{252} \mathrm{Cf}$ source at a fluence of $6.7 \times 10^{10} \mathrm{~cm}^{-2}$. After exposure the samples were examined by a fiber optic probe coupled to a fluorimeter using $355 \mathrm{~nm}$ excitation. The characteristic anthracene fluorescent spectra were observed as shown in Figure 10. The spectral intensity from the irradiated sample (dotted line) was less than half the amplitude of that from the non-irradiated sample (solid line). The irradiated sample was then regenerated by re-exposure to the UV light source and the fluorescent spectrum remeasured. As seen in the figure, the intensity from the regenerated sample (dashed line) was nearly equal to the non-irradiated sample showing that the result of the neutron exposure is reversible.

Figure 11 shows a schematic of a two-photon crossed laser beam optical readout system developed by Cullum et al. [9] consisting of the following components: 1) a Qswitched, frequency doubled Nd-YAG laser, 2) dielectric mirrors which split the beam into two frequencies, $\lambda_{1}$ and $\lambda_{2}, 3$ ) an optical delay stage so that the two beams reach the focal point in the sample simultaneously, 4) an $x-y-z$ translational stage for sample placement, 5) a short-pass filter to reject the excitation frequency, and 6) an intensified charge-coupled device (ICCD) for readout. For reading the anthracene doped PMMA, the frequencies the two beams were the first harmonic of the Nd-YAG laser at $1064 \mathrm{~nm}$ and the second harmonic at $532 \mathrm{~nm}$. As shown in Figure 12, neither of these frequencies was sufficient to cause fluorescence in the sample but simultaneous absorption of a photon from both beams at the position where they crossed resulted in stimulated fluorescence. We believe that the relatively large $(90 \mu \mathrm{m})$ diameter of the spot is due to small imperfections in the polymer caused by heating due to the intensity of the tightly focused $1064 \mathrm{~nm}$ beam spot. It should be possible to reduce this effect by adjusting the intensity of the beams, with the goal of being able to monitor tracks left by protons scattered from individual neutrons. 


\section{Summary and Conclusions}

The effects of ionizing radiation on 3D ORAM materials were investigated using charged particle beams. The experimental results confirmed the theoretical predictions that ORAM materials are sensitive to HCP radiation. These results support the potential use of ORAM materials as radiation detectors/dosimeters since the measurable intensity is proportional to dose over several orders of magnitude. A permanent change was observed in the properties of SP doped PMMA exposed to protons, alpha particles and ${ }^{12} \mathrm{C}$ ions, resulting in a new material, which fluoresces under examination by the CLSM. The fluorescent intensity at a given dose varies with particle type and energy. This property is likely to prove very useful for studies of the effects of radiation exposure to various types of particles and energies.

The fluorescent intensity of the exposed materials was unchanged after several months. This shows that they were observing a new material distinct from the fluorescent Form II of the SP molecule, which is short-lived at room temperature. The absorption and fluorescent spectra were measured and are also distinct from Form II. To date insufficient quantities of the new material have been made for further chemical analysis.

In the range of energies investigated, the incident energy per nucleon can be determined from the width of the depth profile, which depends on the particle range in PMMA. Transport calculations show that the ${ }^{12} \mathrm{C}$ ranges are expected to deviate from the proton and alpha particle ranges at higher energies per nucleon.

These studies were performed at relatively high doses, which were limited on the lowdose side by the capabilities of the particle accelerator and the sensitivity of the detection system. Future efforts should be directed at investigating the effects at lower doses, investigating the effects for other photochromic materials, determining the sensitivity to neutron exposures and investigating other imaging techniques in order to look at the detailed track structure.

Monte Carlo modeling shows that the CLSM fluorescence profiles can be understood in terms of the transformation of the SP molecule to a new fluorescent form as a result of energy deposition due to secondary ion production by the energetic charged particles. The ionization curves from the model are folded with the gaussian response function of the CLSM and multiplied by a function representing the attenuation in the materials. The result is an excellent approximation to the shape of the measured intensity curves.

The properties of the materials observed in this study are very promising since the energy and dose can both be determined from a combination of penetration depth and fluorescent intensity, thus allowing for an accurate calculation of the dose equivalent. The Monte Carlo modeling shows that it is possible to understand the observed depth profiles in terms of the energy deposition in the films and the instrument response. This is an important first step for computer interpretation of the observed track structure, which is necessary for development of an automated reader for a detector/dosimeter system (see [11] for more details.)

The response of 3D ORAM materials to neutrons was investigated as well. We compared two 3D ORAM materials consisting of PMMA doped by the photochromic molecules SP and anthracene, and found that the anthracene doped material was preferable for a practical neutron dosimeter since it was stable in both the non-fluorescent 
and fluorescent forms over a period of several months. Exposure of anthracene doped PMMA in the fluorescent form to neutrons showed a significant reduction in the fluorescent intensity. The fluorescence could be restored by exposure to UV light showing that the process is reversible, a desirable property for a practical dosimeter.

\section{Acknowledgements}

This research is sponsored by the U.S. Department of Energy under Contract DE-AC05960R9464 (NN-20). The contributions of the following are greatly appreciated: A.K. Readshaw, G.O. Brown, R.G. Weiss, N.A. Guardala, J.L. Price and S.C. Mueller. 


\section{References}

[1] A.S. Dvornikov, S.E. Esener and P.M. Rentzepis, Three-dimensional Optical Storage Memory by Means of Two-Photon Interaction, in Optical Computing Hardware, Jahns J. and Lee S.H., Eds., Academic Press Inc., Boston (1994) 287325.

[2] M. Moscovitch, US Patent No. 5,319,80 (1994).

[3] M. Moscovitch, US Patent No. 5,498,876 (1996).

[4] M. Moscovitch and D. Emfietzoglou, Simulation of radiation effects on threedimensional computer optical memories, J. Appl. Phys. 81, 58 (1997).

[5] J. Mobley, J.S. Bogard, M. Moscovitch, and T. Vo-Dinh, Signal expression and initial design for a 3D ORAM radiation dosimeter reader, Proceedings of SPIE 3534, 519-530 (1999).

[6] G.W. Phillips, A.K. Readshaw, G.O. Brown, R.G. Weiss, N.A. Guardala, J.L. Price, S.C. Mueller and M. Moscovitch, Observation of radiation effects in threedimensional optical random access memory for use in radiation dosimetry, Appl. Radiat. Isot. 50, 875-882 (1999).

[7] A.K. Readshaw, G.O. Brown, G.W. Phillips, R.G. Weiss, N.A. Guardala, J.L. Price, S.C. Mueller and M. Moscovitch, Investigation of 3D ORAM Materials for Radiation Dosimetry, Radiation Protection Dosimetry 85, 451-454 (1999).

[8] J. F. Ziegler and J. M. Manoyan, The Stopping Power of Ions in Compounds, Nucl. Instr. and Meth. in Phys. Res. B35, 85-98 (1988), (computer program and additional information available at the following website: http://www.research.ibm.com/ionbeams/home.htm).

[9] B.M. Cullum, J. Mobley, J.S. Bogard, M. Moscovitch, G.W. Phillips and T. VoDinh, Three-dimensional optical random access memory (3D-ORAM) for use as radiation dosimeters, Anal. Chem. 72, 5612-5617, (700).

[10] C. Gonzales and E.C. Lim, Electronic spectra and photophysics of the two stable conformers of anthracene dimer: evaluation of an ab initio structure prediction," Chem. Phys. Lett. 39, 382-388 (700).

[11] M. Moscovitch and G.W. Phillips, Radiation Dosimetry Using ThreeDimensional Optical Random Access Memories, in SSD 2001 Summer School Issue, to be published in Nucl. Instr. and Methods B. 


\section{Figure Captions}

Figure 1. Diagram showing the use of two-photon absorption to excite the SP molecule from the non-fluorescent spyrobenzopyran form to the fluorescent merocyanine form [1, $5,9]$.

Figure 2. Visible beam spots as a result of exposure of films to $2.5 \mathrm{MeV}$ proton beams PMMA films doped with SP at 1\% concentration, except for the rightmost film which was pure PMMA. Exposures were, left to right, 4, 37, 171, 796, and 374 Gy. The SP doped films show increased darkening of the beam spot as the dose increases [6].

Figure 3. CLSM depth profiles for protons on SP doped PMMA. Similar depth profiles are seen for 2 and $5 \mathrm{MeV}$ alpha particles and $6.5 \mathrm{MeV}{ }^{12} \mathrm{C}$ ions. The lines are smooth curves drawn through the data [6].

Figure 4. Full width at half maximum (FWHM) for CLSM depth profiles versus energy per nucleon. The line is a second order polynomial fit to the data [6].

Figure 5. Transport calculations for the range of protons, alphas and ${ }^{12} \mathrm{C}$ in PMMA.

Figure 6. Maximum fluorescent intensities from the CLSM analysis plotted versus dose for the indicated particle and energy. The statistical errors in the intensities are less than the symbol size and the lines are smooth curves fitted to the data.

Figure 7. Chemical structure of the anthracene molecular monomer.

Figure 8. Top view of two possible geometries for the anthracene molecular dimer [10].

Figure 9. Diagram showing two photon excitation of the anthracene molecule.

Figure 10. Fluorescence spectra for anthracene-doped PMMA prepared in the fluorescent monomer form, before irradiation, after neutron irradiation and after regeneration of the irradiated material [9].

Figure 11. Schematic of the two-photon crossed beam readout system for use with 3D ORAM dosimetry [9].

Figure 12. False color image of fluorescence from an anthracene-doped PMMA material using the crossed-beam optical readout system. Red shows little or no fluorescence with intensity increasing toward the blue. The side and bottom graphs show intensity cuts through the center of the image [9]. 


\section{Spirobenzopyran}

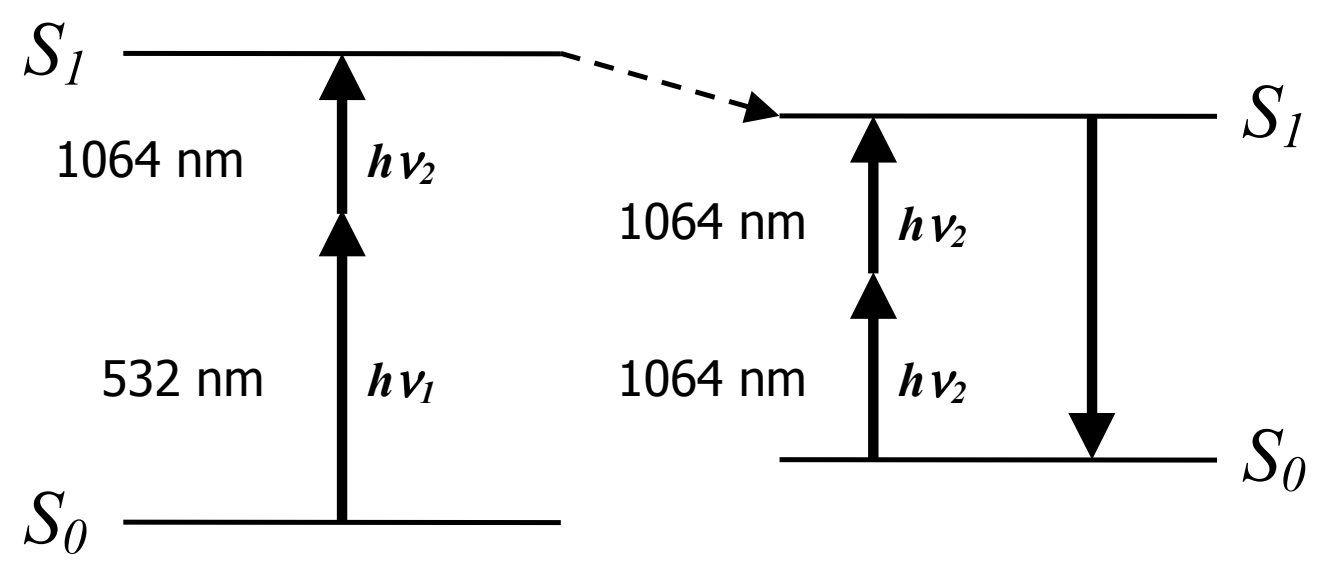<smiles>CN1c2ccc(Cl)cc2C(C)(C)C12C=Cc1cc([N+](=O)[O-])ccc1O2</smiles>

Form I

Spirobenzopyran

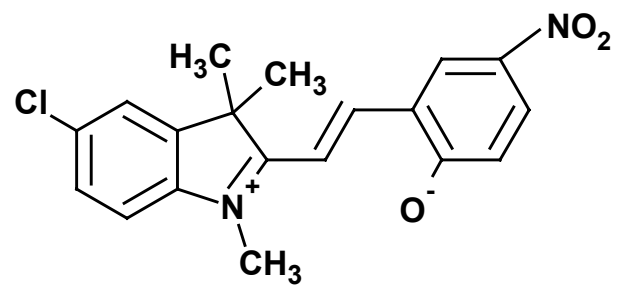

Form II Merocyanine 


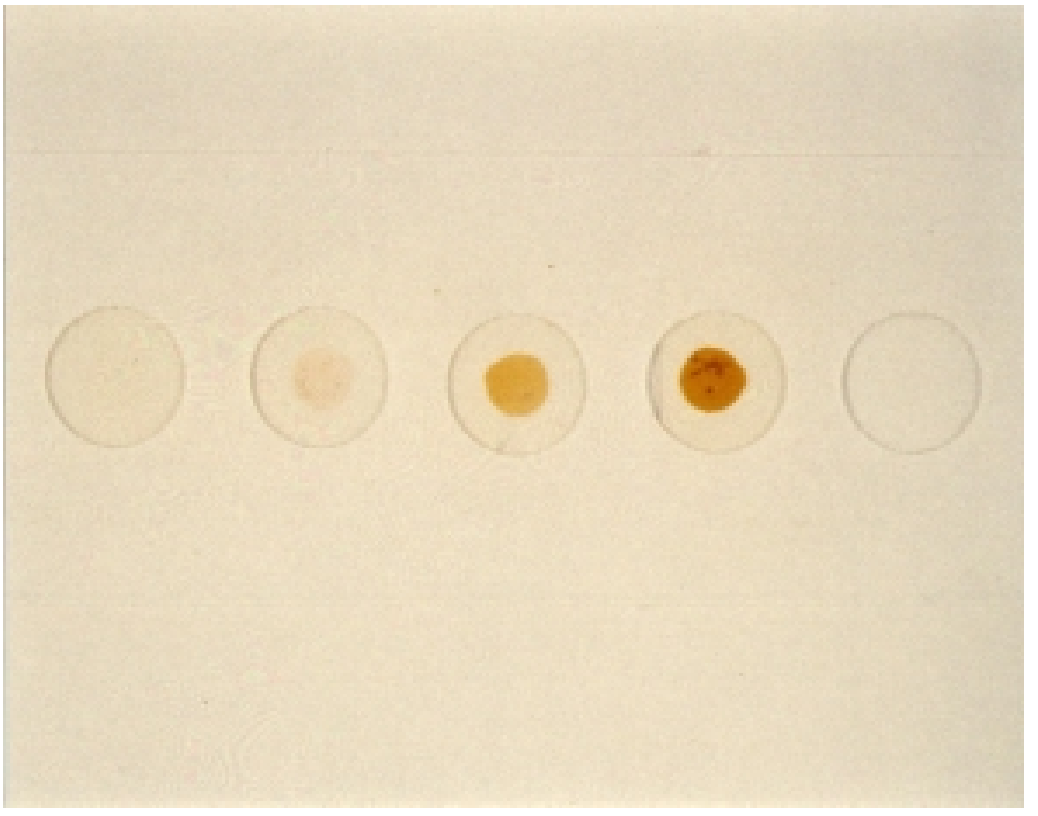

figure 2 


\section{Proton Depth Profiles}

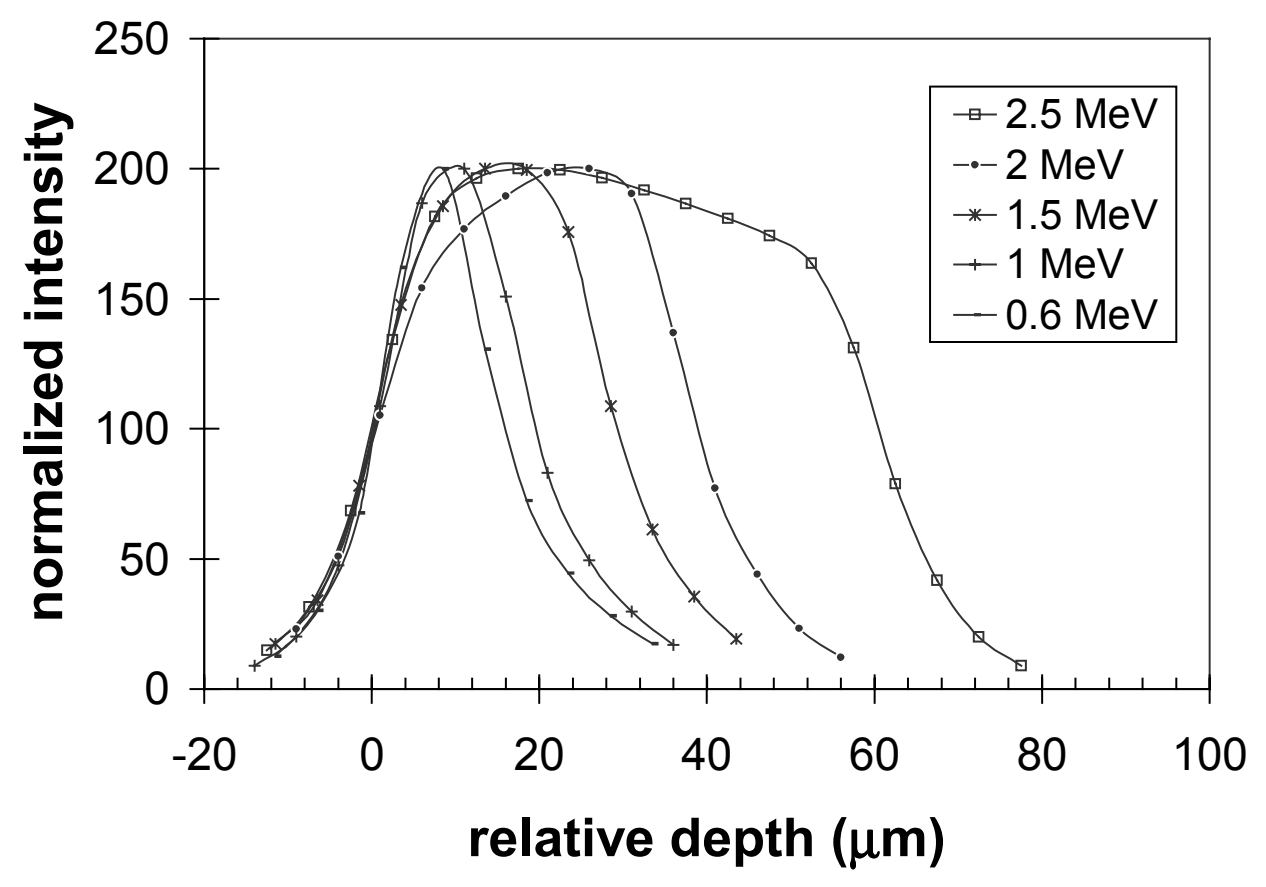


CLSM Depth Profile Widths

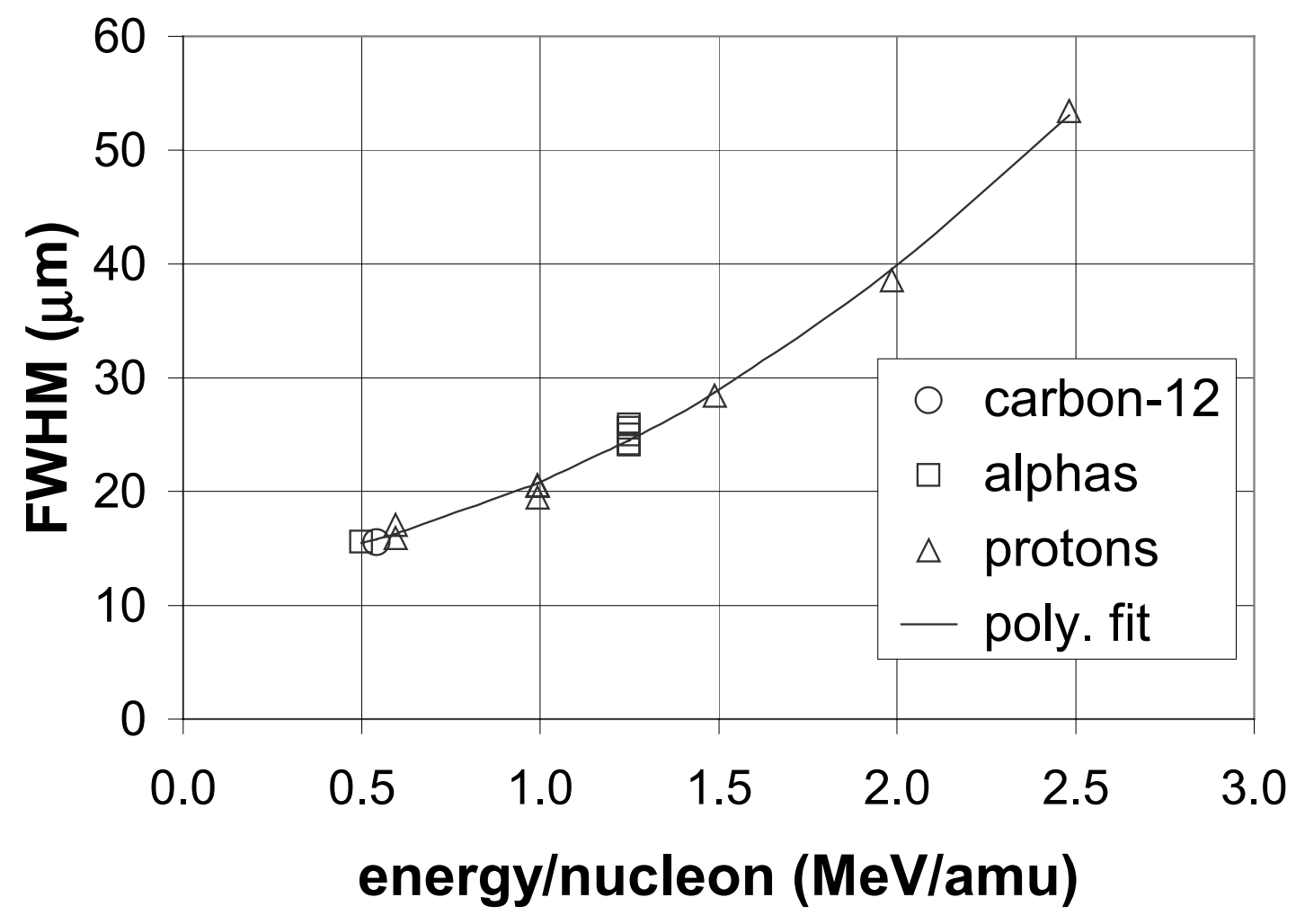




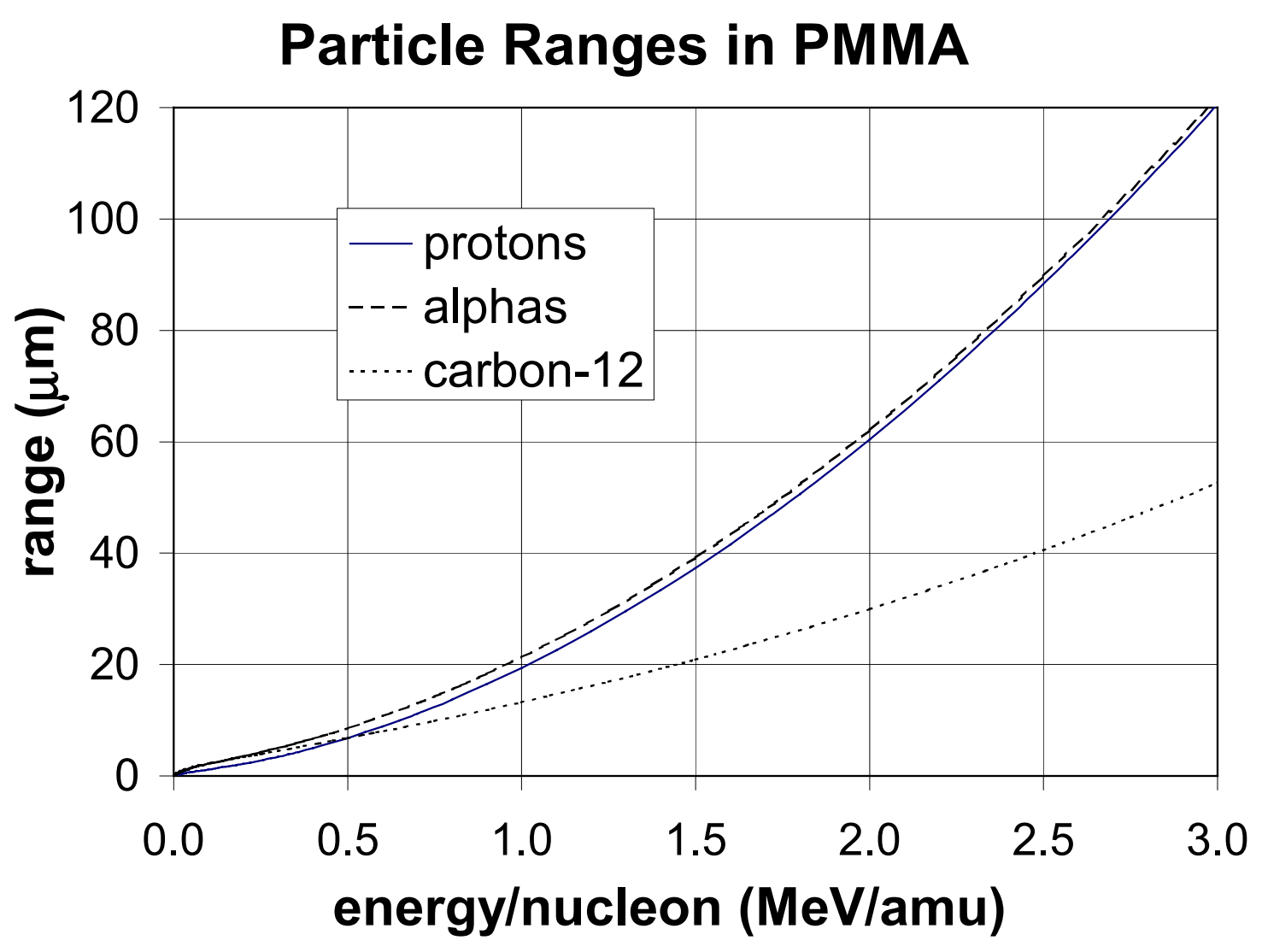


HCPs on SP Doped PMMA

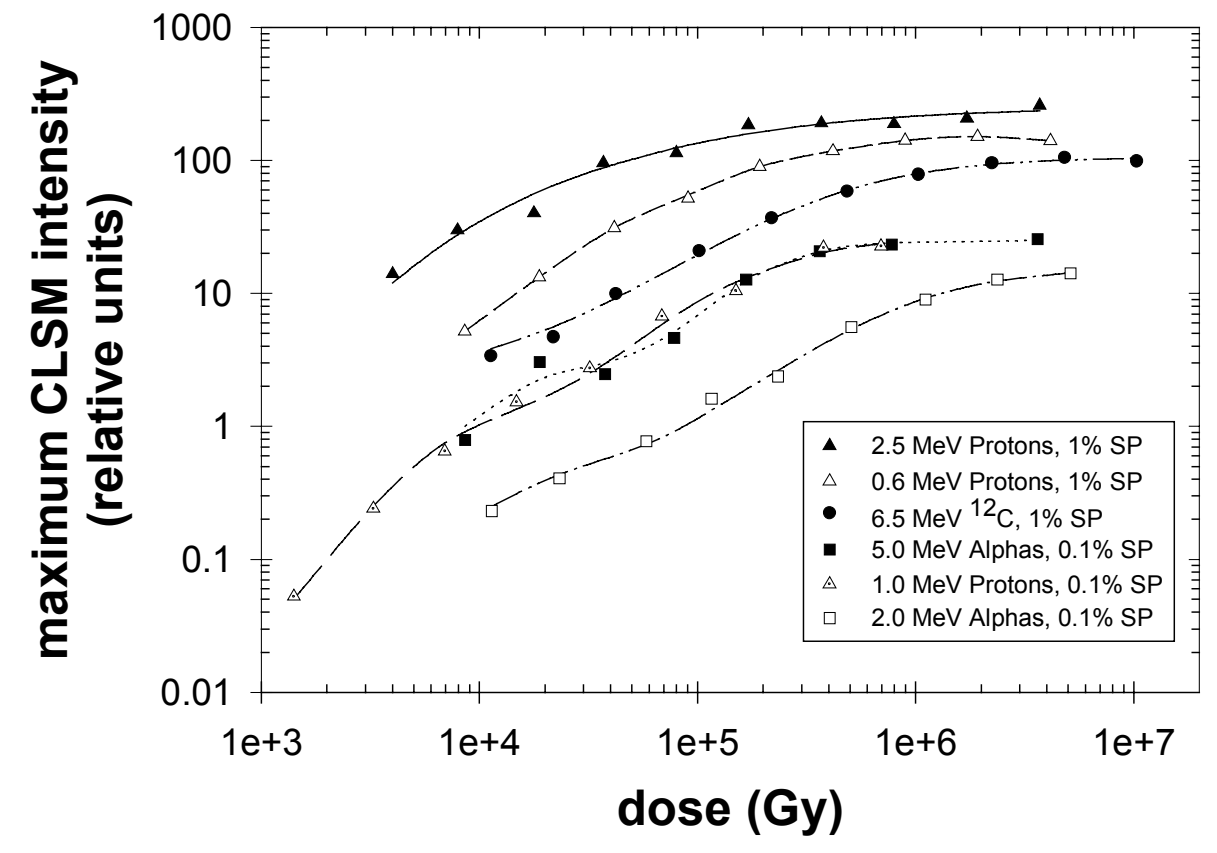




\section{Anthracene Monomer}

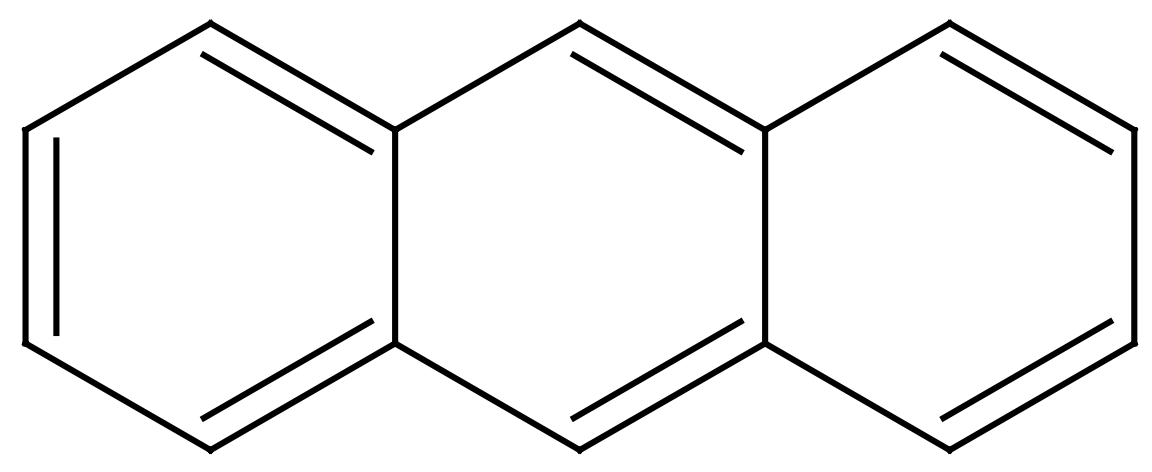




\section{Anthracene Dimer Geometries}

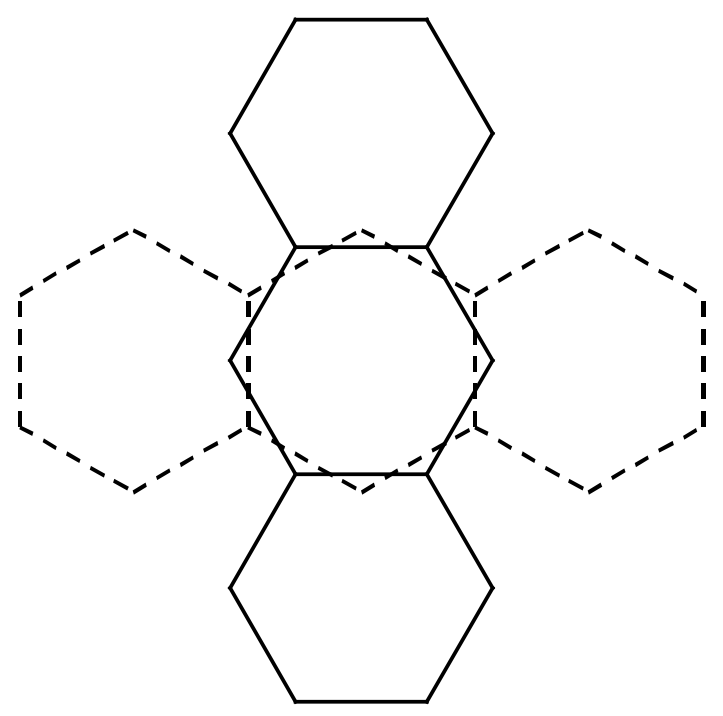

crossed

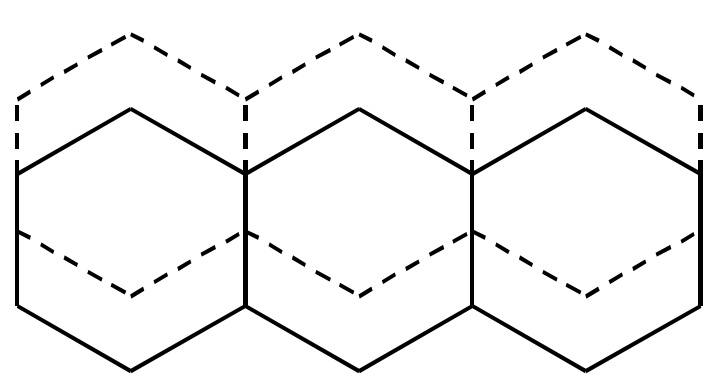

displaced parallel 


\section{Athracene}

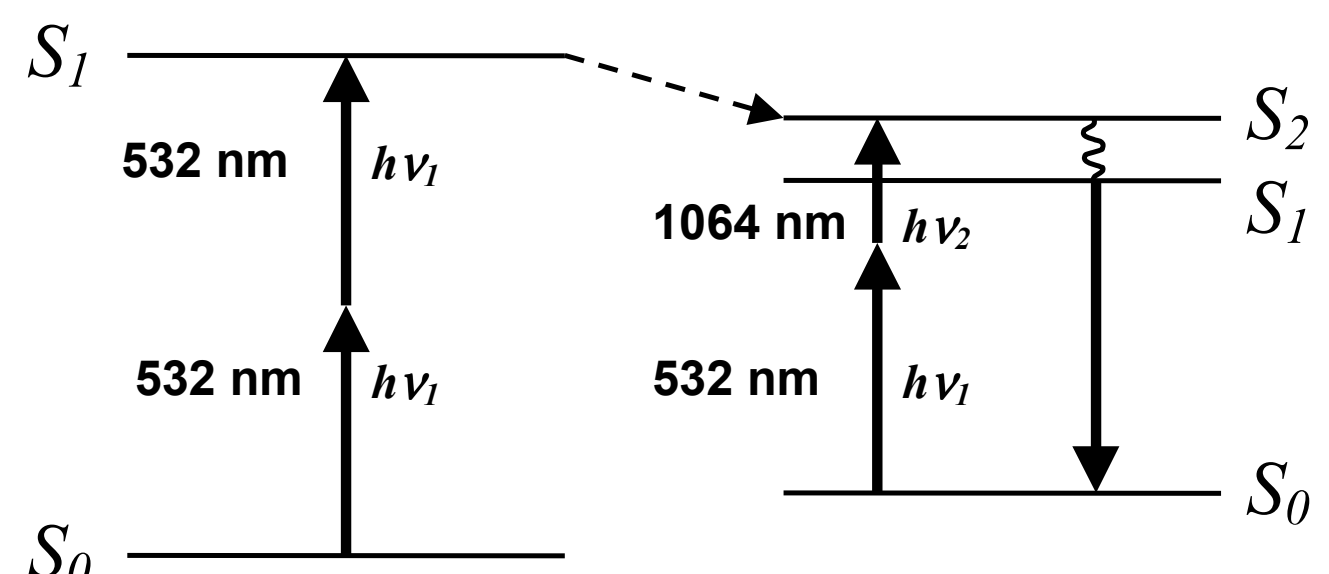

Dimeric Form

Monomeric Form 


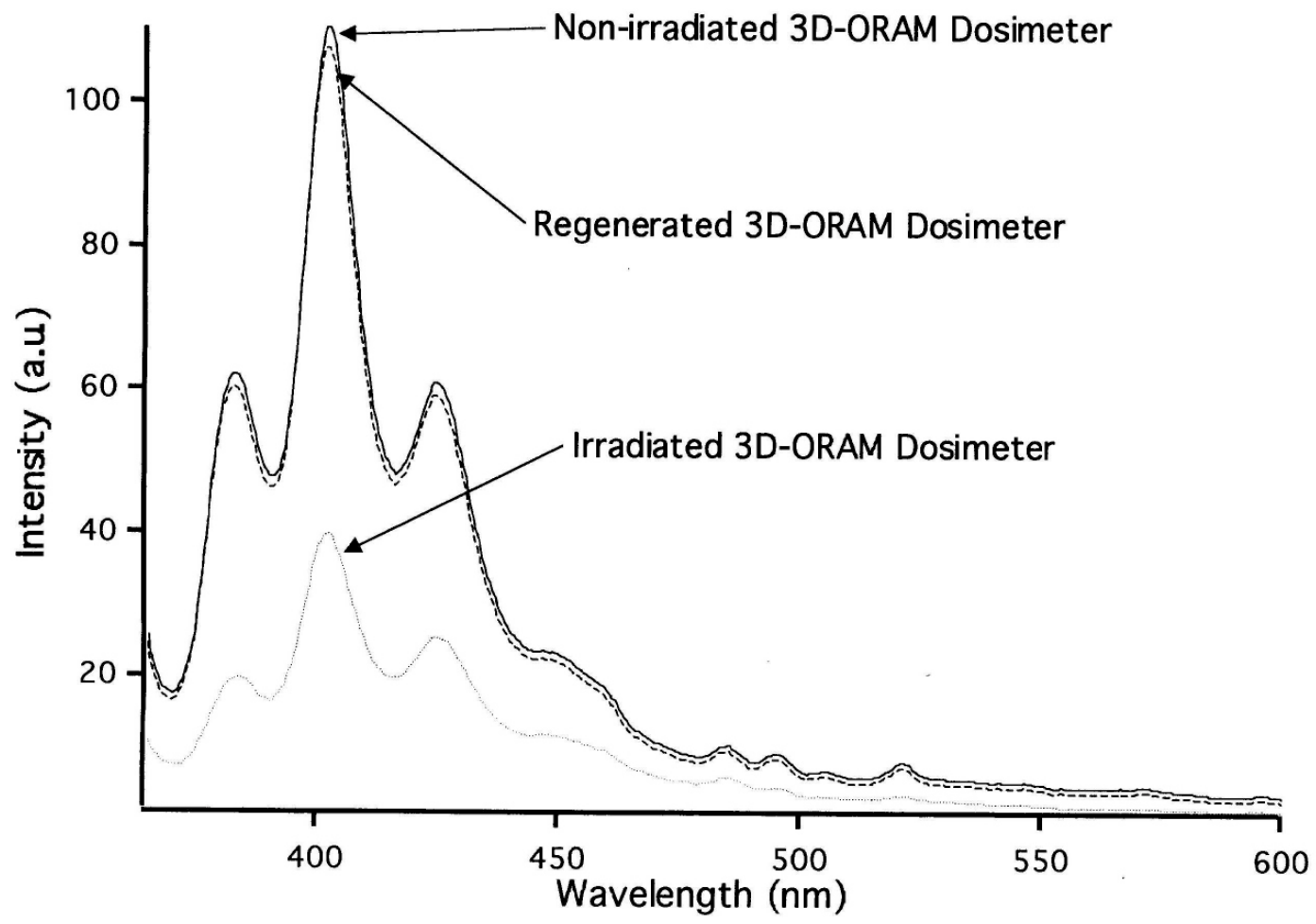




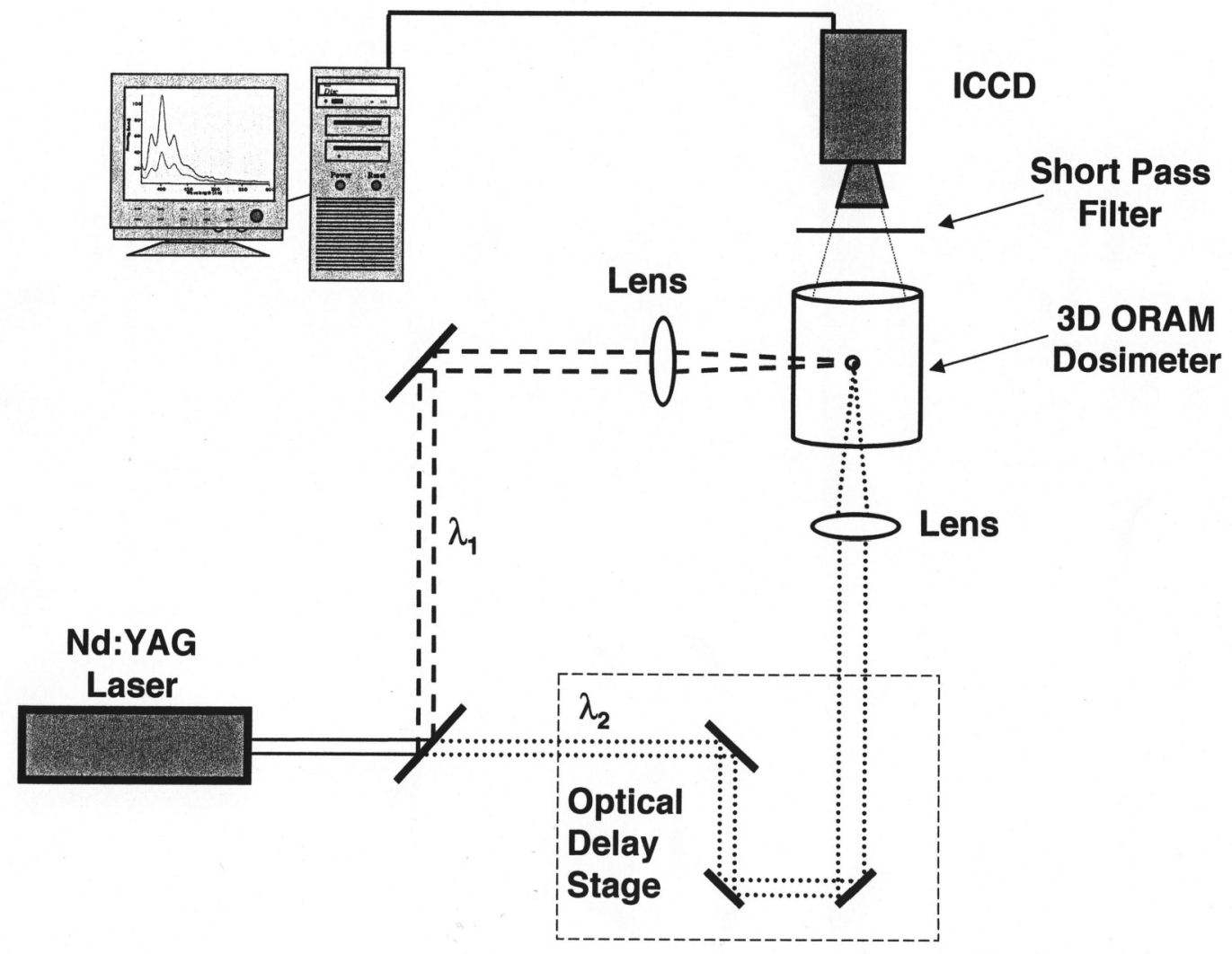




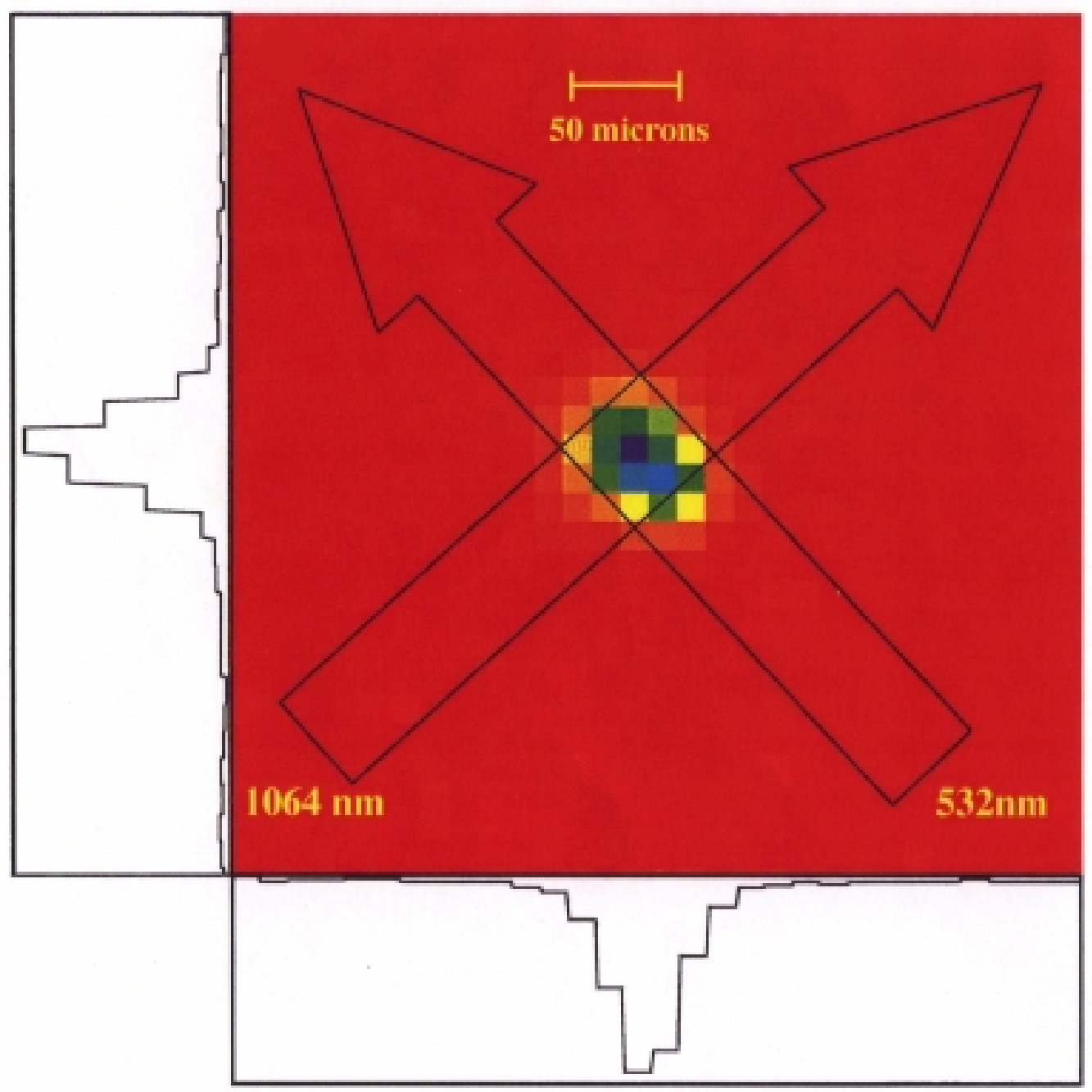

figure 12 